\title{
Childhood Epithelioid Hemangioendothelioma
}

National Cancer Institute

\section{Source}

National Cancer Institute. Childhood Epithelioid Hemangioendothelioma. NCI Thesaurus.

Code C114926.

An epithelioid hemangioendothelioma that occurs during childhood. 